\title{
Ultrasonic Indoor positioning for umpteen static and mobile devices
}

\author{
Schweinzer Herbert, Kaniak Georg \\ Vienna University of Technology, Institute of Electrical Measurements and Circuit Design \\ Gußhausstr. 25/354, A-1040 Vienna
}

\begin{abstract}
Indoor ultrasonic (US) systems for locating mobile devices are presented since years differing in operating modes and various parameters. This paper depicts the alternatives and derives a positioning system, called LOSNUS (Locating of Sensor Nodes by UltraSound), which is directed at supporting the locating of umpteen static devices together with mobile nodes.
\end{abstract}

\section{Introduction}

Indoor locating systems are often used to track paths of mobile devices and/or persons. However, in factory and building automation numerous sensor and actuator devices are often deployed which are connected to a control network or a wireless sensor network (WSN). Primarily being crucial parameters for application tasks, device positions can support important service functions especially if they are gained by a repetitively performed locating process, e.g. maintenance in case of dislocated devices, device integration in a WSN on base of the position, and improvement of network security. In the latter case, device positions can be used for setting up a security concept based on "Location based Keys" [1, 2]. This prevents intruding of an attacker, who pretends being a localized device of a room, into the whole network.

\section{State of the art}

US locating systems are mostly based on "A-B measurements" which are performed between US transmitters (A) and US microphones as receivers (B). The velocity of ultrasound in air is rather slow which allows a cost-effective measurement of the Time-of-Flight (ToF) by means of a fast start signal. Mostly utilizing RF or Infrared, this start information is transmitted synchronously with the US signal and is used for starting the time measurement on the receiver side which is stopped at receiving the US signal. With known sound velocity the ToF can easily be converted into the US path length which, however, is requiring a Line-of-Sight (LOS) condition to get the real distance between $\mathrm{A}$ and $\mathrm{B}$. Based on measuring distances to minimal three transducers with well-defined 3D positions, the position of a device can be calculated by an algorithm named "trilateration" which delivers the three Cartesian coordinates of the device by solving a system of three quadratic equations. If distances to more than three well-defined transducer positions are measured an extended algorithm called "multilateration" allows a device position calculation with improved accuracy e.g. by minimizing the least square error of different solutions of the quadratic equations.

An explicit start signal can also be omitted. In this case, receivers have to store the Time-of-Arrival (ToA) of US signals. If four transducers with well-known positions are minimally included in the locating, three Time-Differences-of-Arrival (TDoA) can be calculated from the four ToAs. This eliminates the need of realizing a start signal but reduces the accuracy of the resulting device position by using a more complicated algorithm names "pseudo-trilateration" or "hyperbolic positioning" for solving the highly nonlinear system of coupled equations. Also here accuracy can be significantly improved by multilateration.

Concepts of US locating systems have to decide a basic alternative: what part of US signal transmission is taken by the localized device, transmitting or receiving? Table 1 shows the main impacts of this decision. At first glance, signal transmission by the device is the easier solution and this type of systems was indeed realized at early systems [3, 4]. In this case, minimal three receivers are mounted in fixed positions of a room and receive the US signal of the localized device in parallel. To enable multiple devices being located, US signal transmissions of devices have to be triggered sequentially by an external coordinator e.g. via RF device addressing [5]. Especially if transmitting uncoded US burst signals, activating of the next device must take care of sufficient decay of US echoes which reduces the number of activated devices within a time interval and thus the activation rate of each device [6]. 
Table 2. Basic alternatives of device locating

\begin{tabular}{|c|c|c|}
\hline & Device transmitting signal & Device receiving signal \\
\hline Effort of device realization & increased & reduced \\
\hline Privacy & No & Yes \\
\hline Device collision avoidance & By using a start protocol & No collisions; broadcast locating \\
\hline Locating rate depending on & Number of devices & Signal coding \\
\hline Well suited for & $\begin{array}{c}\text { Few fast devices or multiple } \\
\text { slow }\end{array}$ & \begin{tabular}{c} 
Numerous devices, fast or slow \\
\hline
\end{tabular}
\end{tabular}

Interestingly, the primary motivation for the alternative system concept which uses transmitters in fixed room positions and only receivers within the devices was that such a system offers "privacy" of the locating process [7]. Since a device does not emit a US signal, it is not possible in this case to supervise device positions by hidden receivers. However, this system type additionally offers significant advantages if multiple devices shall be located in parallel. Locating is performed in a broadcast operation without any collisions at receiver side. The locating rate is primarily influenced by the protocol of transmitter activations and by coding the transmitted signals or not: without coding the locating rate is relatively low because of waiting for sufficient echo decays before activating the next transmitter. A high locating rate demands about simultaneously transmitting of locating signals which have to be coded [7] and a powerful signal detection in case of signal overlapping. Signal overlapping can occur both by a signal of a different transmitter (Multiple-Access-Interference, MAI) or by an overlapping with a short range echo or a multipath transmission (Inter-Symbol-Interference, ISI). Since the signal processing of device locating only intends to detect LoS signals, especially MAI can be reduced significantly by well-chosen transmitter delays. Although at first glance this scheme reduces the locating rate, the transmitted signals can be significantly shorter than in case of parallel sending because of reduced signal overlapping.

\section{System parameters}

Table 2 displays some fundamental parameters of a broadcast system for locating signal receiving devices. The main alternative is given by different needs of mobile or static devices. Primarily, mobile devices need a sufficient fast locating for adequate path tracking. On the other hand, static devices as sensors and actuators often need only a low activation rate and are typically connected over a WSN with low communication speed. However, a deployment of umpteen static devices can create the need of a position accuracy higher than that used for mobile devices. Thus, sufficient locating mobile and static devices with the same equipment can be reached by using broadband transducers with coded signals.

Especially for locating mobile devices the equipment has to be permanently installed. This makes the cost of locating equipment a crucial aspect. On the other hand, the cost of a local US receiver is a crucial aspect for static devices. Minimal demand of local processing power and position calculation by a central location server are adequate methods to reduce the effort of static devices. Additionally, position estimation with TDoA allows also reducing the effort. Since the locating rate of static devices has to be adapted to the activity rate, the locating system has to enable static devices to take part in actual locating cycles at any time. A significant difference between mobile and static devices is mainly the data rate of network communication which typically requires the use of a fast network, e.g. WLAN, instead of a WSN.

Table 1. Fundamental system parameters of a broadcast locating system

\begin{tabular}{|c|c|c|}
\hline & Mobile device & Static sensor / actuator device \\
\hline Locating rate & Medium high, high & Low \\
\hline Positioning accuracy & Low (tracking), presence check & High for device distinguishing \\
\hline Narrowband (burst signals) & Slow, reduced accuracy & Reduced accuracy \\
\hline Broadband (coded signals) & Fast, high accuracy & High accuracy, receiver effort $\uparrow$ \\
\hline Low cost receiver & Local computing of position & Use of a location server \\
\hline Low cost locating equipment & $\begin{array}{c}\text { Permanently } \\
\text { installed }\end{array}$ & \begin{tabular}{c} 
Permanent or removable \\
\hline TDoA instead of ToF
\end{tabular} \\
\hline Network & - & $\begin{array}{c}\text { Lower cost, but reduced } \\
\text { accuracy }\end{array}$ \\
\hline WLAN & WSN \\
\hline
\end{tabular}




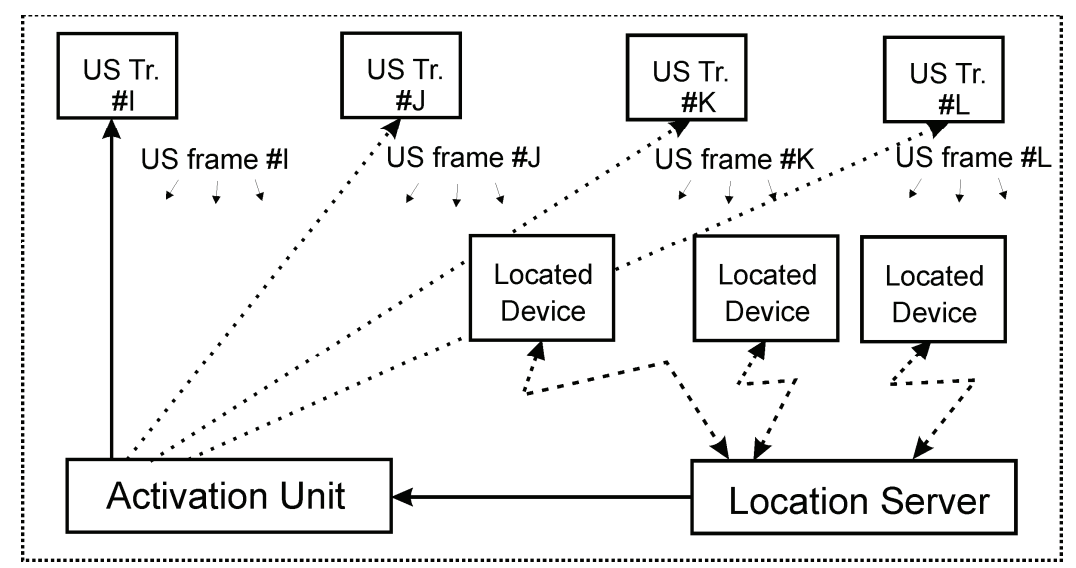

Figure 1. System overview: Sending of US signal frames by the transmitters controlled by the activation unit, transferring of received locating data by the devices to the location server which calculates the device locations

\section{Main characteristics of the locating system LOSNUS}

Mainly directed towards low-cost realization [8, 2], the indoor US system LOSNUS is realized as a centralized system (Fig. 1). One activation unit per room stores the signals for the US transmitters which are installed at defined positions in the room, mostly near to the ceiling. The transmitters are activated sequentially via individual cables. This enables using an activation unit with only one switched signal output channel. Devices which are to be located receive the transmitted signal frames in parallel. Beside of optionally local pre-processing the minimal reactions of a device are storing received data and transferring it via e.g. WSN to the location server. This server is the only unit which has the full information necessary for computing device positions. The used method of calculating the positions is Time-Difference-of-Arrival (TDoA).

The locating process delivers high position accuracy of $\sim 10 \mathrm{~mm}$ (depending on the device position) and robust operation. The locating sequence uses exactly defined delays between frame transmissions whose minimal values are already sufficient that the Line-of-Sight (LoS) signals of consecutive transmitters are not overlapping (Fig. 2). This method eliminates any MAI of the transmitted signals. Thus, only interferences of LoS signals with echoes of former transmissions which are already damped can occur. In this case, the LoS signal will dominate and signal processing will mostly deliver the precise ToA suppressing the echoes. If locating is performed repetitive with a short intermission between the sequences, the start frame will be omitted. Nevertheless, the intermission allows a device to synchronize with the following sequence. The start frame will only be used in a start-stop locating where is has to trigger the next locating cycle.

The transmitters need to be broadband. They are sending frames which contain an identical lead-in frequency, an also identical linearly frequency modulated chirp, and an individual transmitter code (Fig. 3 ). The lead-in frequency gives time for signal rising and allows a roughly checking of being a proper frame. The chirp enables to fix the frame reception time with the full resolution of the sample clock. The transmitter code is individually defined and can be changed by the activation unit. This definition of frame contents allows different levels of local frame interpretation:

- A signal can be recognized by using a level trigger

- The lead-in frequency is an indicator of a frame instead of any disturbance

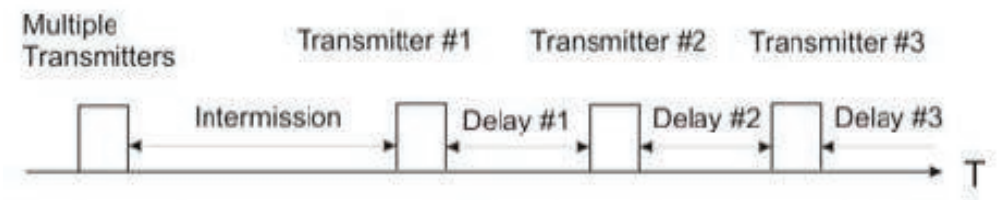

Startframe Frame\#1 Frame \#2 Frame \#3

Figure 2. Sequence of frame transmissions with start-frame (only for single cycle locating with long intermissions) and non-overlapping LoS reception of transmitter identifying frames 


\section{Frame \#x}

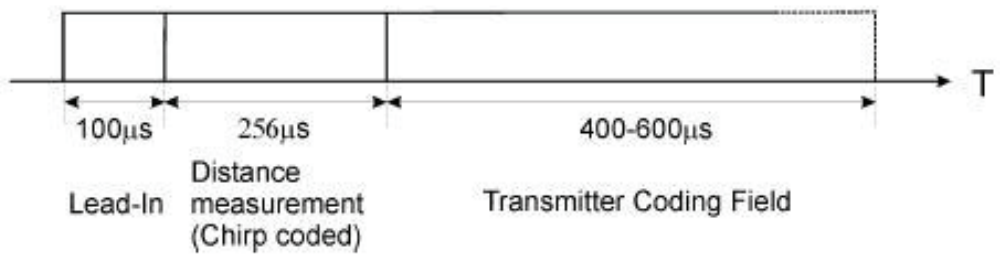

Figure 3. Setup of transmitted frames consisting of lead in, constant chirp linearly frequency modulated, and transmitter coding time slot (used frequency band $35 \mathrm{kHz}-65 \mathrm{kHz}$ ).

- Correlating of the chirp with a locally stored reference defines the precise sample position of the ToA within the frame

- All frame parts which are not interpreted, especially the transmitter coding, have to be stored locally and transmitted after frame reception to the location server

If the transmitter codes are defined as logical codes \#1 to \#n which are used in every locating sequence independently of the actual assignment of physical transmitters, devices are enabled to extract the full frame content "ToA" and "logical transmitter number" (LTN). For this purpose, reference patterns of all logical codes have to be provided for local correlative processing.

\section{Components for locating of devices}

Fig. 4 shows the few additional components of a device necessary for receiving US frames. It is assumed that the device contains a microcontroller with a synchronous serial port and a network interface for transferring stored data to the location server. After amplifying and filtering the microphone input, the signal is converted by a 1-bit converter (polarity converter) realized by a comparator. The signal is sampled by the clocked input of the synchronous shift register. An additionally comparator triggers the potential arrival of a frame with sufficient level. With this signalling a time stamp is captured and the data recording is started. Thus, with stored frame data and based on the time stamp the reception time can be evaluated with the resolution of the local clock. This is done by correlating the data with a chirp reference to get the exact position of the chirp within the frame data. Afterwards the transmitter code being immediately following the chirp can easily be analyzed.

\section{Location server}

Sensor/actuator devices have to transfer the received locating data - pre-processed or stored - via a WSN link to the location server. The location server is a central computer which holds the necessary information for calculating the device positions. Those are mainly the Cartesian coordinates of the transmitters, the used transmitter coding and the sequence of activating the transmitters, and the delay time intervals. Additional information, e.g. constraints of the room layout and dimensions, can aid a successful and robust locating. The calculated device positions will be sent back to the devices. This cannot be seen as a violation of the privacy because the centralized location server is a trusted authority and the communication link should use encrypted data transfers.

To guarantee the consistency of the locating data, any changes of the locating sequence have to be

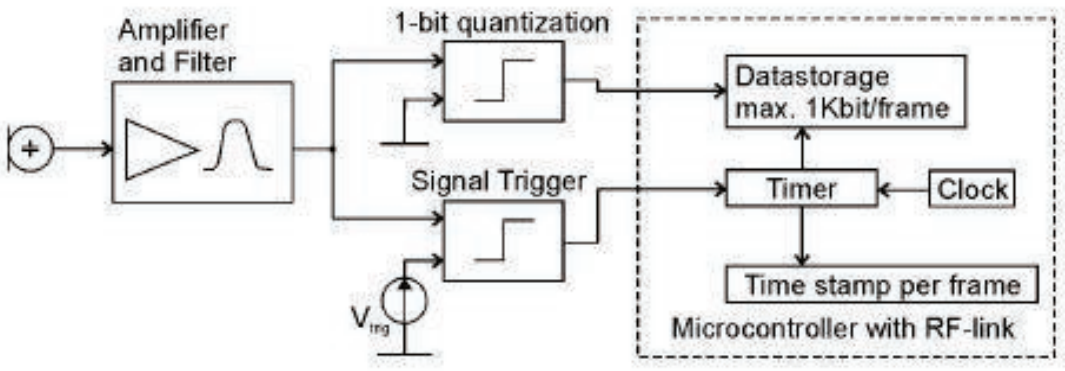

Figure 4. US receiving sensor device with local data storage of frames which are sent after a location cycle by RF link to the locating server: Microcontroller with additional microphone, amplifier and filter, 1-bit quantization of US with $1 \mathrm{Mbit} / \mathrm{s}$, signal trigger for roughly marking the local time of frame reception. 
initiated and recorded by the location server. Moreover, repetitive locating of static devices enables the calculation of mean values of positions with improved accuracy. Also the supervision of static devices is possible by notifying devices which do not take part in locating for some time or which are suddenly dislocated.

In principle, the locating of mobile nodes has to be handled by the location server in a similar manner. However, different additional tasks have to be performed, as e.g. path recording, transferring locating to a neighboring locating system if a mobile device is changing from one room to another, and room access control.

\section{Security aspects}

US locating has the significant advantage that US signals cannot pass walls, closed windows and doors [9]. Considering the security of performing control and supervision tasks in a room, a main attack could be to participate in the WSN communication and spuriously influence it although being outside of the room. A special method of encrypting the local communication can protect against such influences: encryption based on "location based keys" (LBK) [1, 2]. In this case, the communication in a room is only possible between partners who are localized within that room. These devices get their LBKs together with their positions from the location server and are restricted by the LBK encryption method to establish only communication links to partners within the room.

If neighboring locating systems are coded in different manner, the room information is implicitly contained in the received location data. Thus, a device located in a specific room will not get a LBK compatible with that of a different room. An attacker will at the most be able to irritate a local functionality in a room by faking his sensor data and his accurate position, but he will not be able to intrude into the whole network.

\section{Test system}

A test system was set up to show the functionality of the locating system. Fig. 5 shows 6 US transmitters mounted on a wall to enable easier access (usually at the ceiling). The transmitter type is Polaroid 600, an electrostatic type which offers a usable frequency band between about $30 \mathrm{kHz}$ to $70 \mathrm{kHz}$. The locating sequence is activated by a special arbitrary waveform generator (AWG) which allows storing and replaying the whole sequence with a precise timing of $5 \mathrm{MS} / \mathrm{s}$. The AWG additionally allows storing the demultiplexer channel together with the samples. An analog amplifier transforms the AWG signal to an amplitude range from 0 to $400 \mathrm{~V}$ which includes the necessary bias voltage of $200 \mathrm{~V}$ for the electrostatic transmitters. The amplified signal is switched to an actual transmitter by a relay demultiplexer.

Fig. 6 shows a sensor module with a WSN interface which is used for setting up the WSN, realizing

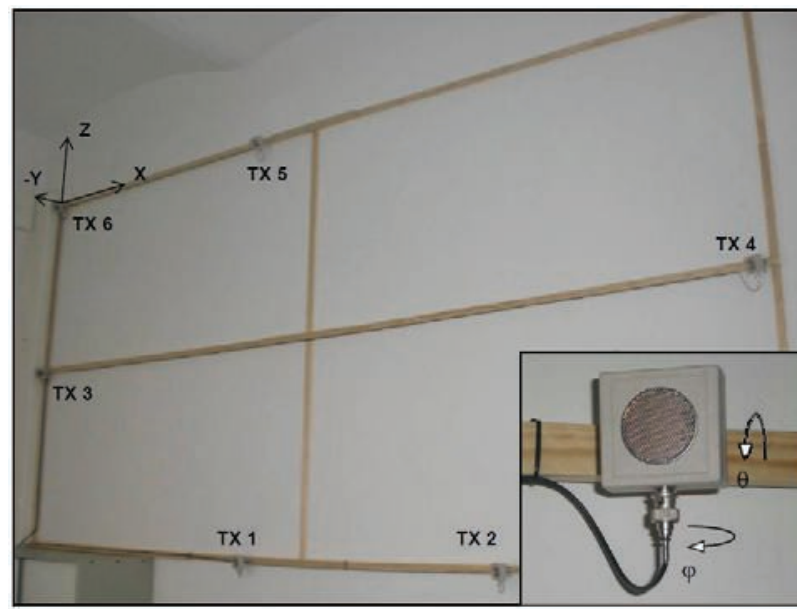

Figure 5. Construction of a frame with 6 US transmitters, for easy operating mounted on a wall; transmitter (type Polaroid 600) turnable in two angles

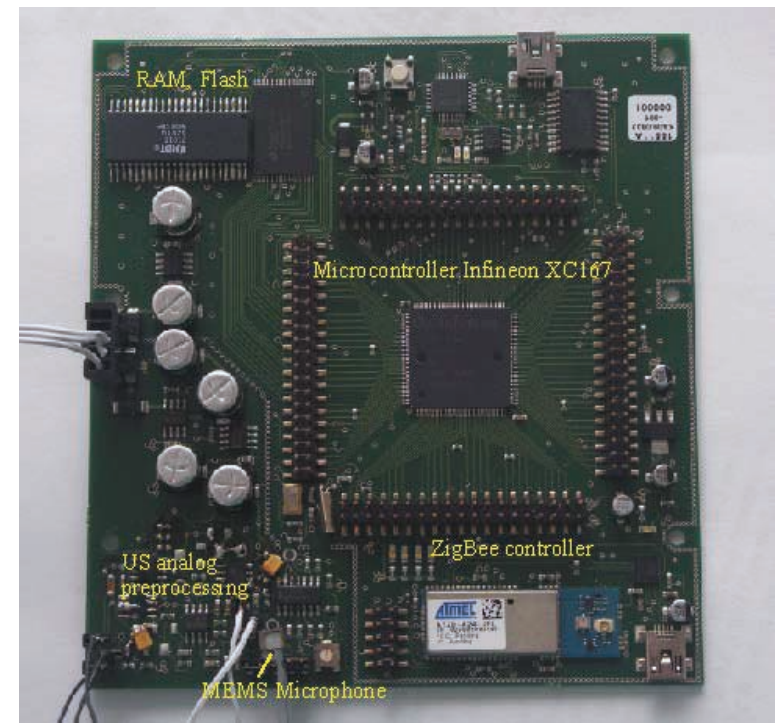

Figure 6. Test board of a generic sensor/actor node for the WSN ZigBee. Main components: Infineon microcontroller XC167, ZigBee controller Atmel ATZBA24-UFLR, external Ram and Flash, US analog preprocessing, MEMS microphone (connected via cable). 
application examples and the basic functionality of the LBK encryption system. The module contains a microcontroller Infineon XC167 with external RAM and Flash, a ZigBee (IEEE 802.15.4) controller Atmel ATZB-A24-UFLR, the necessary US analogue hardware and a US MEMS microphone (Knowles SPM0204UD5). The sample rate of the sensor module is $1 \mathrm{MS} / \mathrm{s}$ which deliver a resolution of time differences of $\pm 0.3 \mathrm{~mm}$.

\section{Conclusion}

This paper gives a short overview over the US locating system LOSNUS. After a presentation of alternative system concepts, LOSNUS features and parameters are discussed which are directed to support both path tracking of mobile devices and periodically supervising of umpteen static sensor/actuator devices in an optimal manner. Testing and developing of additional system features is an ongoing work. Actual focuses are effective methods of calibrating the transmitter positions and realizing the encryption method based on LBK.

\section{References}

[1] Y. Zhang, W. Liu, W. Lou, and Y. Fang, "Location-based compromise-tolerant security mechanisms for wireless sensor networks," IEEE Journal on Selected Areas in Comm., 24(2), pp. 247-260, February 2006.

[2] H. Schweinzer, G. Kaniak. "Ultrasonic device localization and its potential for wireless sensor network security." Control Engineering Practice 18 (2010) 852-862, Elsevier, 2009.

[3] Ward, A., A. Jones, A. Hopper, "A new location technique for the active office," IEEE Personal Comm. Magazine, Vol. 4, No. 5, 1997.

[4] M. Addlesee et al. "Implementing a sentient computing system," IEEE, 34(8), 2001.

[5] N. B. Priyantha, A. Chakraborty, and H. Balakrishnan, "The Cricket location-support system," ACM MobiCom, USA, 2000.

[6] C. Randell and H. Muller. "Low cost indoor positioning system," Ubicomp 2001: Ubiquitous Computing, USA, 2001.

[7] M. Hazas and A.Ward, "A high performance privacy-oriented location system," PERCOM 03: Pervasive Computing and Comm., 2003.

[8] H. Schweinzer, G. Spitzer. "Ultrasonic locating system optimized for low cost, high efficiency and secure application", IEEE IECON, Porto, Portugal, 2009.

[9] N. Sastry, U. Shankar, and D. Wagner. "Secure Verification of Location Claims," ACM Workshop on Wireless Security WiSE'03, San Diego, Cal., USA, 2003. 